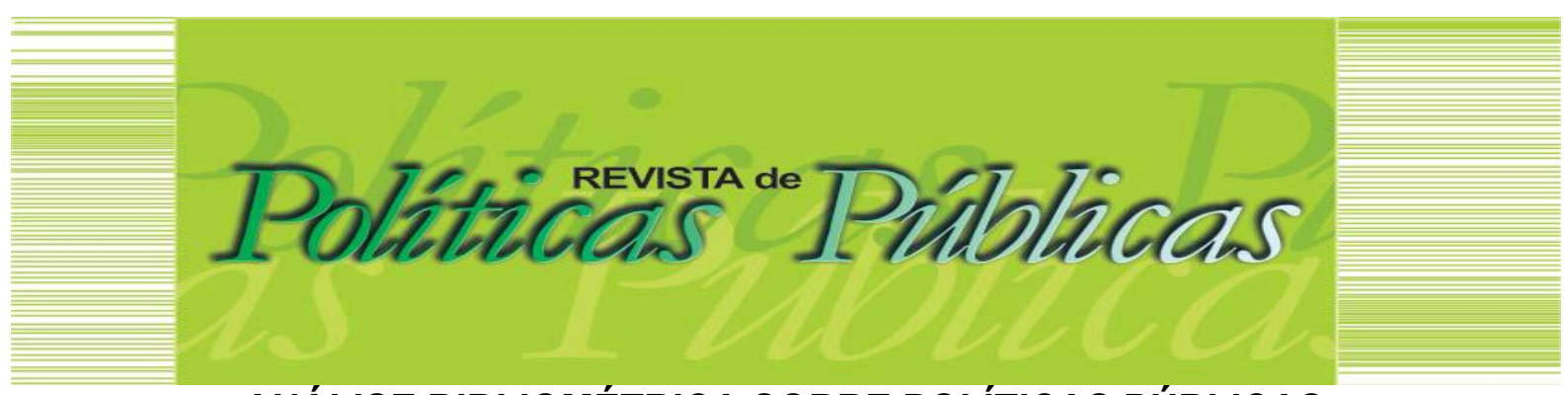

\title{
ANÁLISE BIBLIOMÉTRICA SOBRE POLÍTICAS PÚBLICAS
}

\author{
Fabio Freitas da Silva ${ }^{1}$ \\ Geísa Pereira Marcilio Nogueira² \\ Ítalo de Oliveira Matias ${ }^{3}$ \\ Ludmila Gonçalves da Matta ${ }^{4}$ \\ Aldo Shimoya 5
}

\section{Resumo}

Este trabalho tem como objetivo mostrar a evolução do tema políticas públicas no contexto mundial e nacional por meio de uma análise bibliométrica. Faz um levantamento de indicadores mais gerais na avaliação global, como o histórico temporal das publicações e os países que mais debatem sobre o tema; enquanto na conjuntura brasileira traz indicadores mais específicos, tais como a rede de colaboração do Brasil com outros países e instituições. Os resultados mostraram que a primeira publicação sobre o tema estudado surgiu em 1909, no cenário mundial, e em 1987 no Brasil; os Estados Unidos destacaram-se na pesquisa com maior número de publicações. Apesar do desenvolvimento tardio na área de políticas públicas, o Brasil ocupa o quarto lugar no ranque mundial. Em relação às instituições, a USP concentra o maior volume de trabalhos publicados, no entanto, Estados Unidos ganha no todo.

Palavras-chave: Bibliometria. Políticas Públicas. Indicadores.

\section{BIBLIOMETRIC ANALYSIS OF PUBLIC POLICIES}

\begin{abstract}
This work aims at showing the evolution of the public policy theme in a national and worldwide context by means of a bibliometric analysis. More general indicators on the global assessment were proposed, such as the timeline of publications and countries that most debate the theme; while in the Brazilian conjuncture, more specific indicators were generated, such as the partnership network between Brazil and other countries and institutions. The main results showed that the first publication in the world about the theme under study was in 1909, while in Brazil it was in 1987. They also demonstrated that the United States stood out relating to the number of publications. Another question was that, despite the late development of the area of public policies, Brazil ranks fourth worldwide. Regarding institutions, Universidade de São Paulo-USP (Sao Paulo University) concentrates the largest amount of published works, however, the United States stands out as a whole.
\end{abstract}

Keywords: Bibliometry. Public Policies. Indexes.

Artigo recebido em: 04/03/2019 Aprovado em: 12/09/2019

DOI: http://dx.doi.org/10.18764/2178-2865.v23n2p754-770.

\footnotetext{
1 Engenheiro de Produção. Doutorando em Planejamento Regional e Gestão da Cidade. Mestre em Engenharia de Produção pela Universidade Candido Mendes. E-mail: fabio1_freitas@hotmail.com

2 Engenheira de Produção. Doutoranda em Planejamento Regional e Gestão da Cidade. Mestra em Engenharia de Produção pela Universidade Candido Mendes. . E-mail: isamarcilio@yahoo.com.br

3 Bacharel em Ciência da Computação. Pós-Doutorado em Engenharia e Ciências dos Materiais pela Universidade NorteFluminense. Doutor em Engenharia Civil. Mestre na COPPE em Engenharia de Sistemas e Computação pela Universidade Federal do Rio de Janeiro. Professor titular da Universidade Candido Mendes. E-mail: italo@ucam-campos.br

4 Cientista Social. Doutora em Sociologia Política. Universidade Candido Mendes. Mestre em Políticas Sociais pela Universidade Federal Fluminense. Professora do Mestrado e Doutorado em Planejamento Regional e Gestão da Cidade da Universidade Cândido Mendes. E-mail: ludmatta@yahoo.com.br

5 Agrônomo. Doutor em Genética e Melhoramento. Professor na Universidade Candido Mendes. E-mail: aldoshimoya@yahoo.com.br
} 
Fabio Freitas da Silva, Geísa Pereira Marcilio Nogueira, Ítalo de Oliveira Matias, Ludmila Gonçalves da Matta e Aldo Shimoya

\section{INTRODUÇÃO}

As principais balizas do debate sobre políticas públicas no Brasil se ancoram na questão da maior ou menor intervenção do Estado, tema suscitado, principalmente, pela atuação dos Governos Lula e Dilma nas políticas sociais.

Souza (2006) informa que a política pública, enquanto área de conhecimento e disciplina acadêmica, nasceu nos Estados Unidos da América (EUA), rompendo, de certo modo, com os padrões tradicionais europeus de estudos e pesquisas voltados para a análise de teorias explicativas sobre 0 Estado e suas instituições. Nos EUA, essa área surgiu orientada para os estudos sobre a ação dos governos. Já no caso do Brasil, Castro (2008) salienta que as práticas das primeiras políticas públicas surgiram no governo de Getúlio Vargas (1930 a 1945) e estavam fortemente relacionadas às questões trabalhistas. A autora ainda destaca o forte caráter excludente das políticas propostas nesse período, em parte pelo fato de tais políticas estarem focadas em grupos e/ou categorias profissionais específicas. E que somente a partir da década de 1980, com o fim da ditadura, foram previstas e propostas as políticas públicas universalistas, como a criação do Sistema Único de Saúde.

Em análise sobre a construção do campo da política pública, Souza (2006) destaca quatro importantes teóricos: H. Laswell, H. Simon, C. Lindblom e D. Easton. Segundo a autora, cada um deles contribuiu significativamente com a formulação da política pública como área de conhecimento. Laswell (1936) introduziu a análise de política pública conciliando o meio científico e acadêmico com a produção empírica dos governos. Simon (1957) lançou a ideia da racionalidade limitada dos agentes públicos, seja por falta de informação, seja por falta de tempo, por exemplo, para a tomada de decisão, e contrapôs esse fato à necessidade do uso da própria racionalidade para modelar o comportamento das decisões públicas. Lindblom (1959; 1979) propôs a incorporação de outras variáveis na análise de políticas públicas, como as relações de poder e a integração entre as diversas fases do processo decisório, destacando a função das eleições, dos partidos e dos grupos de interesse, por exemplo. Easton (1965) definiu as políticas públicas como um sistema que recebe entradas e, por conseguinte, produzem saídas influenciadas por essas entradas.

Tendo em vista essa diversidade de pensamentos, torna-se laborioso conceituá-la à medida que se observam autores com uma gama diversa de definições, as quais se complementam, inclusive. Não obstante, no campo da ciência política, existem, pelo menos, duas definições clássicas sobre as políticas públicas. A primeira diz respeito à necessidade de ser uma política elaborada para enfrentar um problema público, constituindo algo que compromete tanto o Estado como a sociedade, não sendo, portanto, sinônimo de política estatal (FREY, 2000) e sim uma política em que o Estado participa como um agente importante (CASTRO, 2008). Já a segunda é mais ampla e desvincula o 


\section{ANÁLISE BIBLIOMÉTRICA SOBRE POLÍTICAS PÚBLICAS}

conceito de política pública exclusivamente do setor estatal, embora o Estado continue exercendo 0 papel central. Nessa percepção, as políticas governamentais são um subgrupo das políticas públicas. E estas, por sua vez, podem ser estabelecidas por organizações privadas, indivíduos, organizações não governamentais, organismos multilaterais, entre outros.

Nesse contexto, percebe-se que a discussão em torno das políticas públicas e do seu papel na construção da cidadania diferem de acordo com o meio social e com a época em questão. Portanto, vimos emergir, nos últimos anos, o crescente interesse do campo científico em análise e estudos sobre políticas públicas, principalmente, em razão da crescente disputa pelos recursos do Estado no gasto social, o que tem suscitado também estudos que se direcionam a entender a influência dos atores envolvidos na elaboração e na condução dessas políticas.

Com o crescente aumento do volume de produção científica, está cada vez mais difícil para os pesquisadores acompanharem a literatura relevante em suas áreas. Os métodos bibliométricos permitem uma avaliação sistêmica, transparente e reprodutível da literatura, mapeando o campo de pesquisa e, assim, orientando o pesquisador para os trabalhos mais influentes (ZUBIC; CATER, 2015). Diante do que foi exposto, o objetivo do presente trabalho foi realizar uma bibliometria sobre o tema políticas públicas a fim de evidenciar o crescimento de trabalhos nessa temática no Brasil e no mundo. Para isso, o artigo utiliza métodos bibliométricos de análise de desempenho e mapeamento.

\section{REFERENCIAL TEORICO}

\subsection{Bibliometria}

Segundo Guedes e Borschiver (2005, p 1), "[...] a bibliometria é um conjunto de leis e princípios empíricos que contribuem para estabelecer os fundamentos teóricos da Ciência da Informação". Ou, em uma abordagem mais clássica, bibliometria é toda pesquisa que tenta mensurar os processos de comunicação da escrita, seja por métodos matemáticos, seja estatísticos (PRITCHARD, 1969).

Existem dois principais usos dessa técnica, uma para análise de desempenho e outra para mapeamento da ciência. A primeira busca avaliar grupos de atores científicos, como países, universidades e pesquisadores, enquanto a segunda tem como intuito revelar a estrutura dinâmica da pesquisa científica ou representar o arranjo cognitivo de uma área de pesquisa. (COBO et al., 2011).

Segundo Zubic e Carter (2015), os cinco principais métodos de mapeamentos bibliométricos são: Análise de citação, cocitação, acoplamento bibliográfico, coautoria e copalavras. Os três primeiros usam medidas de influência e similaridade construídas com base nos dados de citações. 
Fabio Freitas da Silva, Geísa Pereira Marcilio Nogueira, Ítalo de Oliveira Matias, Ludmila Gonçalves da Matta e Aldo Shimoya

O penúltimo mede a colaboração por meio dos dados de coautoria e o último busca conexões entre palavras encontradas nos títulos, palavras-chave ou resumos dos documentos. A Tabela 1 mostra 0 resumo desses métodos.

Tabela 1 - Resumo dos métodos bibliométricos apresentados

\begin{tabular}{|c|c|c|c|}
\hline $\begin{array}{l}\text { Método } \\
\text { Unidade de análise }\end{array}$ & Descrição & Prós & Contras \\
\hline $\begin{array}{l}\text { Citação } \\
\text { (Documento, Autor, } \\
\text { Periódico) }\end{array}$ & $\begin{array}{l}\text { Estima a influência de } \\
\text { documentos, autores ou } \\
\text { periódicos por meio de taxas de } \\
\text { citação. }\end{array}$ & $\begin{array}{l}\text { Pode encontrar rapidamente os } \\
\text { trabalhos importantes no } \\
\text { campo. }\end{array}$ & $\begin{array}{l}\text { Novas publicações têm menos } \\
\text { chances de serem citadas. } \\
\text { Portanto, a citação como medida } \\
\text { da influência é tendenciosa para } \\
\text { publicações mais antigas. }\end{array}$ \\
\hline $\begin{array}{l}\text { Cocitação } \\
\text { (Documento, Autor, } \\
\text { Periódico) }\end{array}$ & $\begin{array}{l}\text { Conecta documentos, autores } \\
\text { ou revistas com base no número } \\
\text { de ocorrências conjuntas em } \\
\text { listas de referência. }\end{array}$ & $\begin{array}{l}\text { É o método bibliométrico mais } \\
\text { utilizado e validado. } \\
\text { Liga documentos, autores ou } \\
\text { revistas com cocitação. }\end{array}$ & $\begin{array}{l}\text { A cocitação é realizada em artigos } \\
\text { citados, logo não é ideal para } \\
\text { mapear frentes de pesquisa } \\
\text { (research fronts). }\end{array}$ \\
\hline $\begin{array}{l}\text { Acoplamento } \\
\text { Bibliográfico } \\
\text { (Documento, Autor, } \\
\text { Periódico) }\end{array}$ & $\begin{array}{l}\text { Conecta documentos, autores } \\
\text { ou revistas com base no número } \\
\text { de referências compartilhadas. }\end{array}$ & $\begin{array}{l}\text { Não requer citações para } \\
\text { acumular. Pode ser usado para } \\
\text { encontrar campos emergentes } \\
\text { e subcampos menores. }\end{array}$ & $\begin{array}{l}\text { Só pode se usado por prazos } \\
\text { limitados } \\
\text { (até cinco anos). } \\
\text { É difícil saber se as publicações } \\
\text { mapeadas são importantes ou não. }\end{array}$ \\
\hline $\begin{array}{l}\text { Coautor } \\
\text { (Autor) }\end{array}$ & Conecta as coautorias & $\begin{array}{l}\text { Mostra as evidências de } \\
\text { colaboração e a estrutura } \\
\text { social do campo. }\end{array}$ & $\begin{array}{l}\text { A colaboração nem sempre é } \\
\text { reconhecida com a coautoria. }\end{array}$ \\
\hline $\begin{array}{l}\text { Copalavra } \\
\text { (Palavra) }\end{array}$ & $\begin{array}{l}\text { Conecta palavras quando elas } \\
\text { aparecem no mesmo } \\
\text { documento. }\end{array}$ & $\begin{array}{l}\text { Usa o conteúdo do artigo para } \\
\text { análise. }\end{array}$ & $\begin{array}{l}\text { Palavras podem aparecer com } \\
\text { diferentes formas e significados. }\end{array}$ \\
\hline
\end{tabular}

Fonte: Adaptado de Zubic e Carter (2015).

Existem algumas ferramentas que executam esses tipos de análise rede, e, dentre elas, destaca-se o VOSviewer, que é um software para construir e visualizar mapas bibliométricos; este é disponível gratuitamente, www.vosviewer.com, para a comunidade acadêmica. Existe um pouco mais de 500 publicações que utilizam essa ferramenta em vários campos temáticos.

As seguintes funcionalidades do software podem ser resumidas segundo Van Eck e Waltman, (2018):

- Criação de mapas baseados em rede de dados, o VOSviewer pode ser usado para mapear as relações entre pesquisadores, organizações, países, palavras-chave ou termos. Estes termos podem ser conectados por coautoria, coocorrência, citação, acoplamento bibliográfico ou links de cocitação. As redes são elaboradas com dados das bases Web of Science, Scopus, Dimensões, PubMed, RIS ou Crossref JSON.

- O software mostra três tipos de visualização: em rede, sobreposição e densidade. Os detalhes dos mapas podem ser explorados por meio da função de zoom, rolagem e pesquisa.

\section{Visualização de redes}

Neste tipo de representação os itens (pesquisadores, organizações, periódicos...) são representados pelos seus nomes/rótulos e por um círculo. O tamanho tanto do rótulo quanto do círculo 


\section{ANÁLISE BIBLIOMÉTRICA SOBRE POLÍTICAS PÚBLICAS}

é determinado pelo seu peso. Alguns itens podem não ser exibidos; isso ocorre para evitar sobreposições. A cor do item é definida pelo cluster ao qual o item pertence. As linhas são os links entre os itens, por padrão são exibidas no máximo 1000 conexões que representam os links mais fortes (VAN ECK; WALTMAN, 2018).

\section{Visualização de sobreposição}

Segundo os autores esta visualização é muito similar à representação de redes, no entanto, os itens são coloridos de forma diferente. Esta coloração é atribuída pela pontuação de um item (ex.: data, citação, fator de impacto, etc..), as cores são em uma escala contínua, variam de menor a maior intensidade.

\section{Visualização de densidade}

Segundo Van Eck e Waltman (2018), esse tipo de representação pode ser aplicado ao item ou ao cluster. Os rótulos são exibidos de maneira semelhante à visualização de rede e sobreposição, porém sem o círculo. Neste tipo de mapa, é atribuída uma cor aos pontos que indicam a densidade do mesmo; essa densidade é definida pelo número de itens no entorno e o peso dos itens vizinhos. A cor azul indica menor densidade e a cor amarela maior densidade.

\section{METODOLOGIA}

A pesquisa foi realizada em duas etapas: a primeira considera o panorama internacional; a segunda aborda somente o Brasil. Na primeira etapa o trabalho apresentou uma visão bibliométrica descritiva (análise de desempenho) sobre a evolução das políticas públicas no mundo, avaliando seu crescimento ao longo dos anos, os principais países que mais publicaram sobre este tema, bem como suas instituições e veículos de comunicação. E, no segundo momento, foi empregada a análise de redes bibliométricas, nas quais os seguintes tipos de técnicas foram utilizados para sua elaboração: Coautoria (autores, organizações, países); Cocitação (autores); Copalavras (palavras-chave dos autores). A metodologia para o mapeamento teve como base os autores Zubic e Cater (2015) que propõem os seguintes passos: projeto de pesquisa; compilação dos dados bibliométricos; análise de dados; visualização; e interpretação.

A base de dados adotada foi a Scopus, disponível no portal periódicos da Capes; essa é a maior base de dados multidisciplinar, possui mais de 71 milhões de registros que incluem, por exemplo, revistas cientificas - pouco mais de 23.700 periódicos revisados por pares -, livros e anais de congresso (ELSEVIER, 2018). Os termos de pesquisa empregados foram: "Public Policy" OR "Public Policies" OR "Public Policie". Buscaram-se os artigos que contivessem estes termos no título, resumo ou palavraschaves, limitando-se a busca àqueles trabalhos publicados em periódicos. 0 período temporal foi do 
Fabio Freitas da Silva, Geísa Pereira Marcilio Nogueira, Ítalo de Oliveira Matias, Ludmila Gonçalves da Matta e Aldo Shimoya

mais antigo disponível na base até 31 dezembro de 2018. Os dados referentes aos documentos encontrados pela pesquisa (periódicos, autores, referencias, citações, etc.) foram armazenados em um arquivo de texto para análise de mapeamento e desempenho. 0 mapeamento foi realizado pelo software bibliométrico VOSviewer versão 1.6 .9 e a análise de desempenho por meio da ferramenta Microsoft Excel®.

\section{RESULTADOS E DISCUSSÃO}

Os resultados foram divididos em duas subseções: a primeira refere-se à análise de desempenho; a segunda ao mapeamento bibliométrico.

\subsection{Análise de desempenho}

Em um panorama global, a primeira publicação sobre políticas públicas no mundo ocorreu em 1909, enquanto, no Brasil, em 1987 (Figura 1). Em ambos os contextos, há um período em que o tema não era muito discutido pela comunidade acadêmica; no mundo, esse período foi de 1909 a 1969 e, no Brasil, de 1987 a 2004. Após essa lacuna temporal, observou-se uma tendência de crescimento sobre o tema.

Figura 1 - Número de publicações por ano.

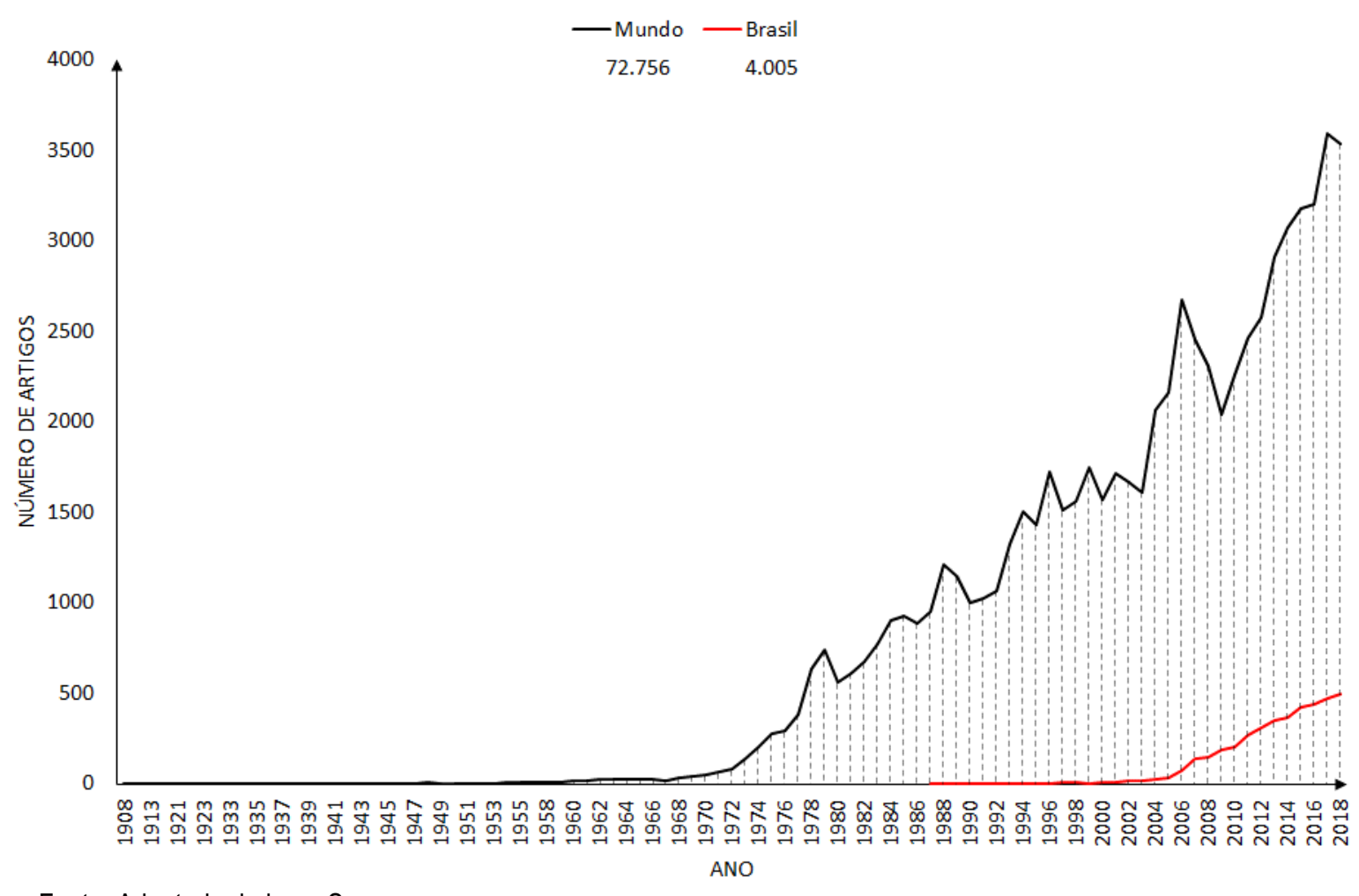

Fonte: Adaptado da base Scopus. 


\section{ANÁLISE BIBLIOMÉTRICA SOBRE POLÍTICAS PÚBLICAS}

A Figura 2 mostra os dez países que mais publicaram sobre o tema políticas públicas. Destaca-se os Estados Unidos com 25.099 trabalhos ao longo dos anos, seguidos pelo Reino Unido, Brasil, Canadá, Austrália e França, com mais de duas mil publicações. É interessante notar que o Brasil apareceu em terceiro lugar, apesar de iniciar o debate sobre o tema somente em 1987.

Figura 2 - Número de publicações dos 10 principais países.

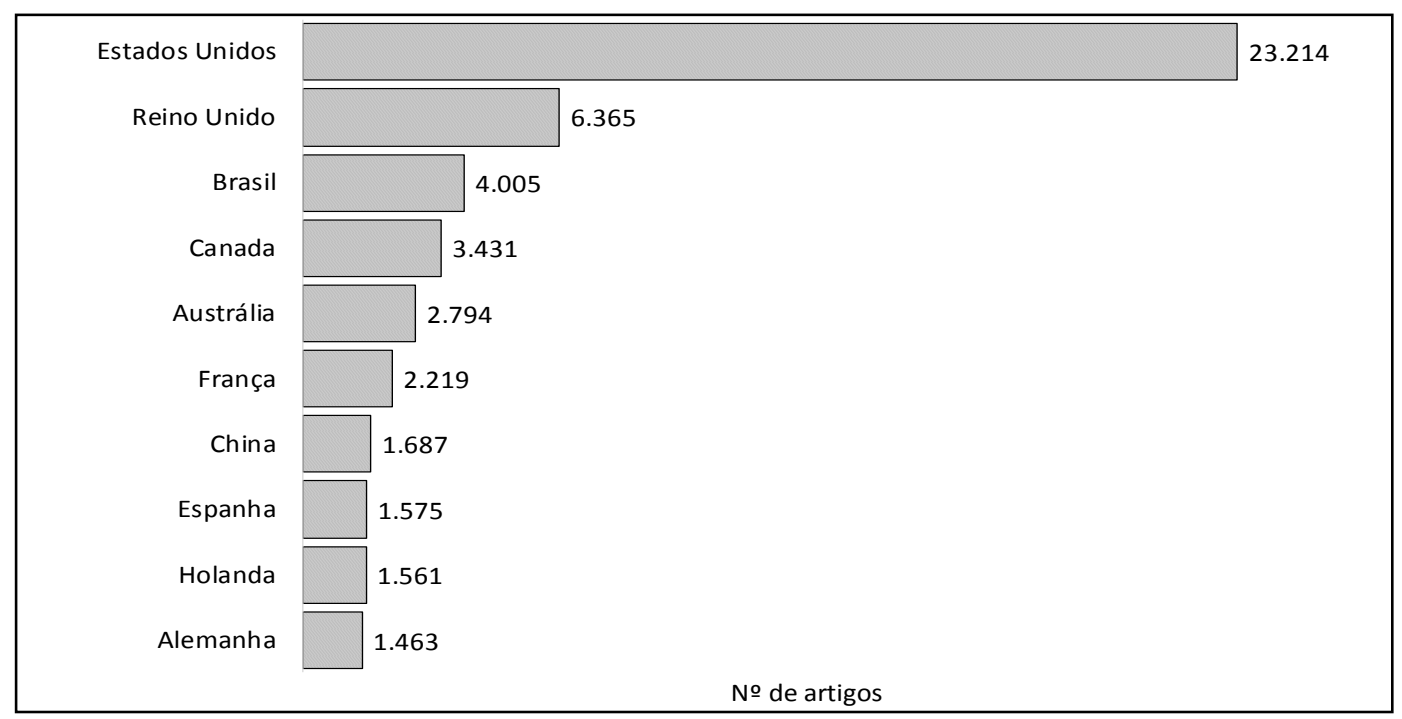

Fonte: Adaptado da base Scopus.

A Figura 3 mostra as 20 instituições que mais publicaram sobre o tema pesquisado. A Universidade de São Paulo (USP) foi a organização que mais produziu artigos científicos no mundo. Destaca-se, também, outra organização brasileira, a Fundação Oswaldo Cruz, que apareceu em décimo segundo lugar. Em contraponto, Estados Unidos ganha em número de trabalhos (Fig. 2), todavia suas publicações são divididas e balanceadas entre as instituições do país.

Figura 3 - Número de publicações das 20 primeiras instituições

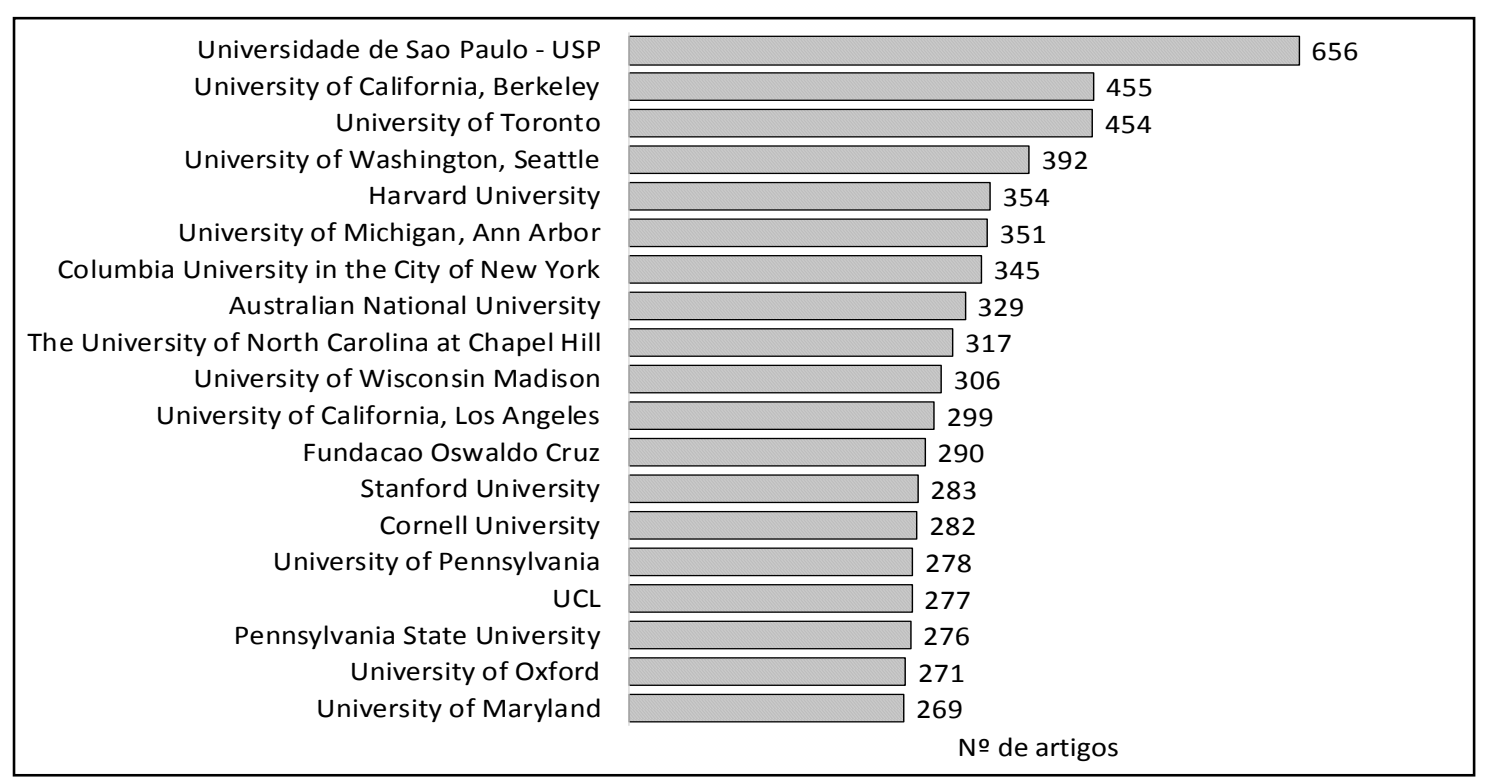

Fonte: Adaptado da base Scopus. 
Fabio Freitas da Silva, Geísa Pereira Marcilio Nogueira, Ítalo de Oliveira Matias, Ludmila Gonçalves da Matta e Aldo Shimoya

$\mathrm{Na}$ Figura 4, se visualizam as diferentes subáreas do tema políticas públicas. Se observa que os campos das Ciências Sociais (25\%) e da Medicina (18\%) concentram $43 \%$ dos trabalhos sobre políticas públicas, seguidos por outros setores menos expressivos. Vale ressaltar que foram encontradas 28 áreas de conhecimento no todo, porém não foram tão expressivas quanto as destacadas na Figura.

Figura 4 - Concentração das publicações por área de conhecimento

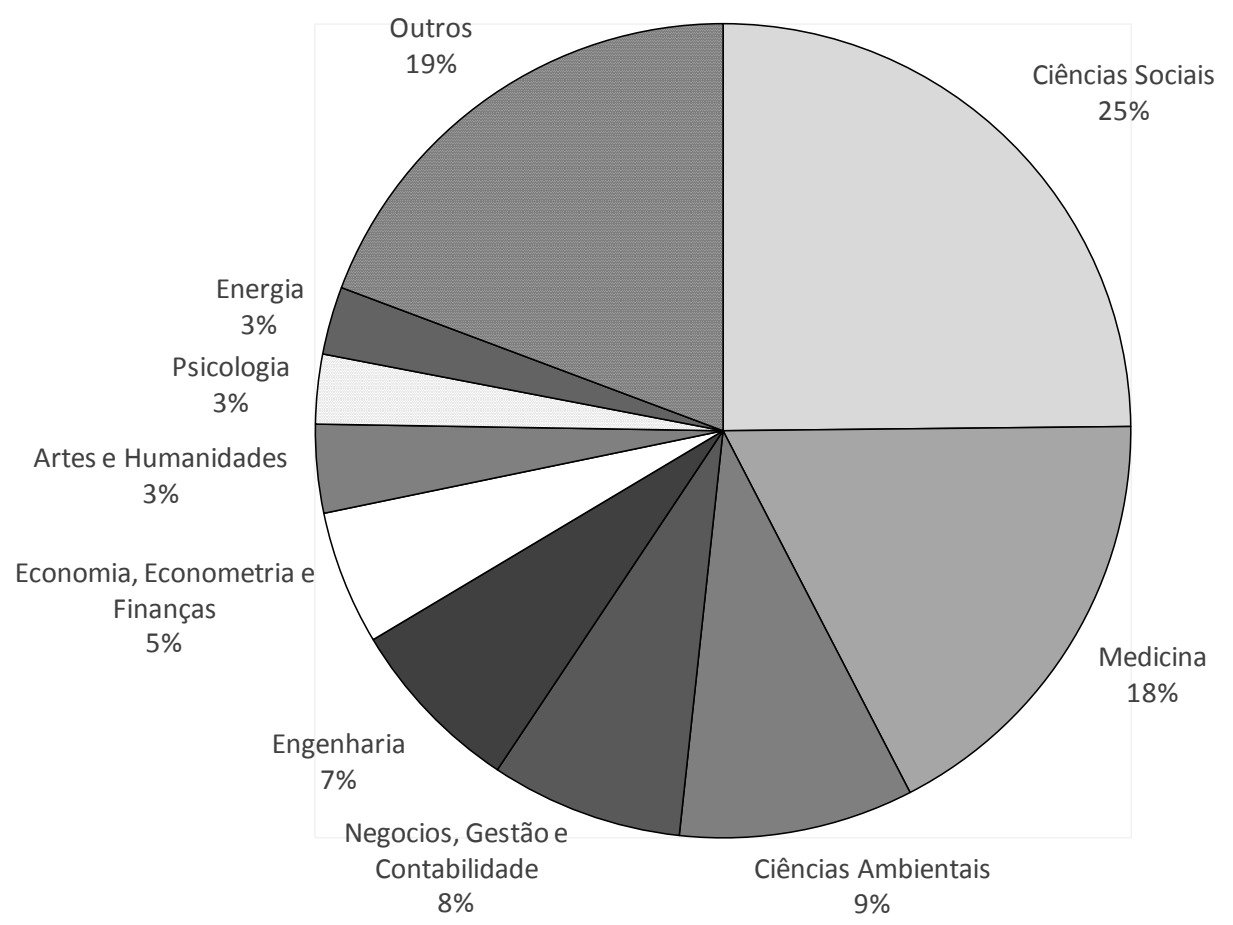

Fonte: Adaptado da base Scopus

Os principais veículos de comunicação (periódicos) do tema estudado estão apresentados na Tabela 2. No total, foram encontrados 157 periódicos, sendo listados os dez primeiros. Pode-se observar um periódico brasileiro, Ciência e Saúde Coletiva, inserido no panorama mundial.

Tabela 2 - Principais veículos de comunicação.

\begin{tabular}{lc}
\hline Periódicos & Publicações \\
\hline Journal Of Policy Analysis And Management & 785 \\
Energy Policy & 661 \\
Telecommunications Policy & 422 \\
Journal Of Cleaner Production & 414 \\
Annual Review Of Population Law & 398 \\
Transportation Research Record & 363 \\
Ciencia E Saude Coletiva & 302 \\
Research Policy & 260 \\
Development In Practice & 256 \\
Journal Of Policy Analysis And Management & 785 \\
\hline
\end{tabular}

Fonte: Adaptado da base Scopus. 


\section{ANÁLISE BIBLIOMÉTRICA SOBRE POLÍTICAS PÚBLICAS}

Na Tabela 3 são exibidos os 10 trabalhos mais citados. Os artigos que tiveram o maior número de citações foram: Teece (1986), com 4.413, seguido por Mozaffarian et al. (2015) com 3.920; os outros tiveram por volta de 1.700 a 2.732 citações. De forma geral, foi possível notar uma predominancia das áreas de Ciências Sociais e Medicina.

Tabela 3 - Trabalhos mais citados sobre políticas públicas no mundo.

\begin{tabular}{|c|c|c|c|}
\hline Autor & Titulo & Citação & Ano \\
\hline Teece, D.J. & $\begin{array}{l}\text { Profiting from Technological Innovation: Implications for Integration, } \\
\text { Collaboration, Licensing and Public Policy }\end{array}$ & 4413 & 1986 \\
\hline Mozaffarian, D. et al. & $\begin{array}{l}\text { Heart Disease and Stroke Statistics-2015 Update : A Report from the } \\
\text { American Heart Association }\end{array}$ & 3920 & 2015 \\
\hline Mcleroy, K.R. et al. & An Ecological Perspective on Health Promotion Programs & 2732 & 1988 \\
\hline Bronfenbrenner, $\mathrm{U}$. & $\begin{array}{l}\text { Ecology of the Family as a Context for Human Development. Research } \\
\text { Perspectives }\end{array}$ & 2494 & 1986 \\
\hline $\begin{array}{l}\text { Kraut, } R \text {. } \\
\text { et al. }\end{array}$ & $\begin{array}{l}\text { Internet Paradox: A Social Technology That Reduces Social Involvement } \\
\text { and Psychological Well-Being? }\end{array}$ & 2086 & 1998 \\
\hline Zhao, $Q$ et al. & A survey of dynamic spectrum access & 2032 & 2007 \\
\hline Easterly, W et al. & Africa's growth tragedy: Policies and ethnic divisions & 2031 & 1997 \\
\hline $\begin{array}{l}\text { Rimm, E.B. } \\
\text { et al. }\end{array}$ & Vitamin E Consumption and the Risk of Coronary Heart Disease in Men & 2000 & 1993 \\
\hline Tilman, $D$ et al. & Forecasting agriculturally driven global environmental change & 1921 & 2001 \\
\hline Stampfer, M.J et al. & Vitamin E Consumption and the Risk of Coronary Disease in Women & 1796 & 1993 \\
\hline
\end{tabular}

Fonte: Adaptado da base Scopus.

\subsection{Mapeamento bibliométrico}

A Figura 5 mostra a rede de colaboração dos países quanto ao tema estudado, e foram selecionadas as nações que possuem no mínimo 5 publicações em coautoria com o Brasil, o que totalizou 24 nações. Tendo como nó central o Brasil, foi possível observar suas conexões com os demais países. Destaca-se uma relação forte - indicado pela proximidade dos itens, quanto maior o número de coautores maior será a similaridade - entre Estados Unidos e Reino Unido com o Brasil; por outro lado, essa relação foi pouco articulada com outros países. Ao observar os grupos formados fica clara a articulação brasileira com Portugal, Espanha e França. A formação desses grupos significa que os países têm uma maior sinergia, ou seja, os trabalhos publicados apresentaram uma combinação dos países de seu respectivo agrupamento com uma maior frequência. 
Fabio Freitas da Silva, Geísa Pereira Marcilio Nogueira, Ítalo de Oliveira Matias, Ludmila Gonçalves da Matta e Aldo Shimoya

Figura 5 - Estrutura colaborativa do Brasil com outros países.

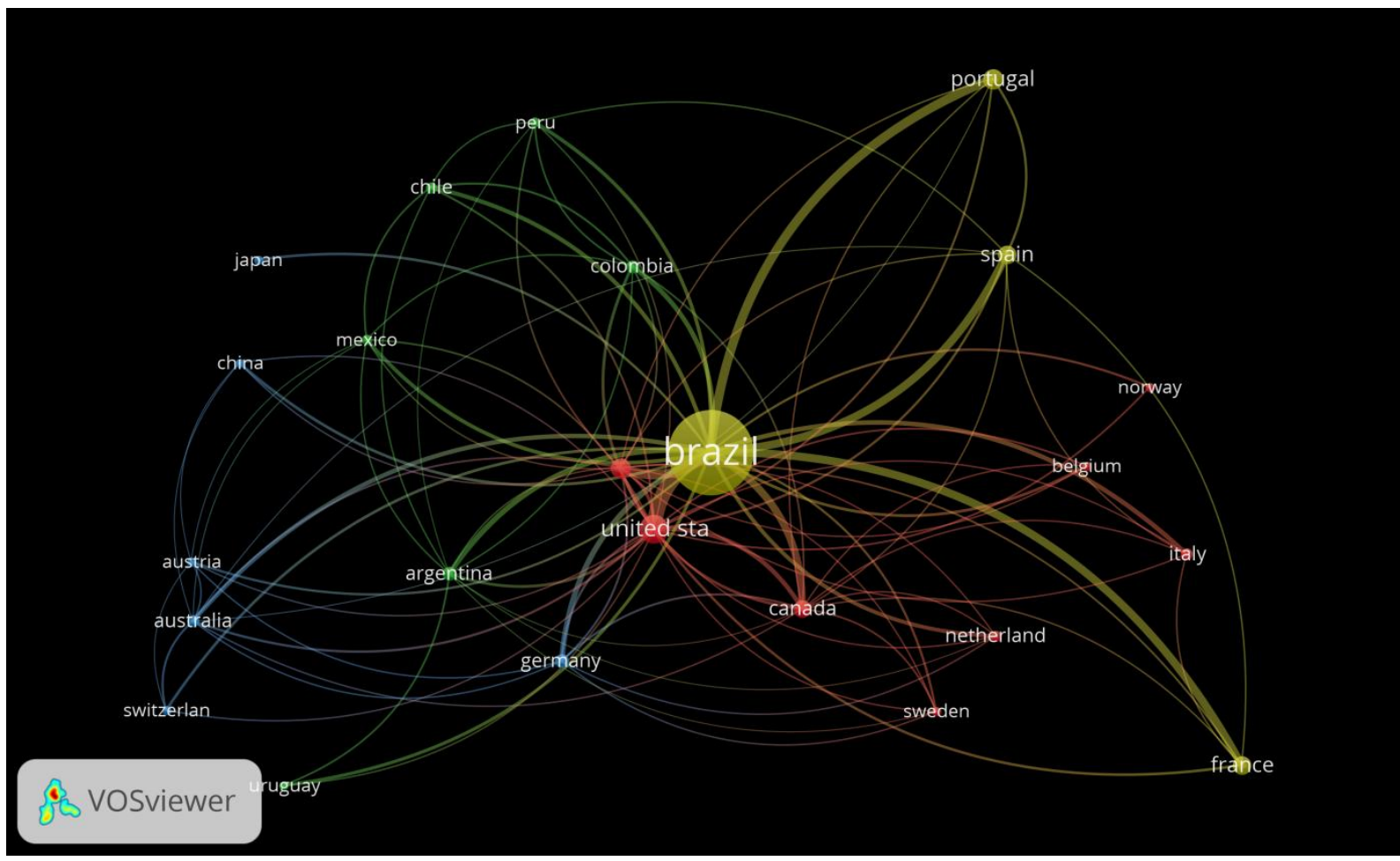

A Figura 6 mostra a rede de colaboração e o mapa de densidade das instituições de ensino; foram selecionadas aquelas com mais de 5 artigos que apresentaram coautoria com outras organizações, o que resultou em 25 instituições. Na parte superior da Figura, foi possível observar a estrutura de participação das organizações, seis grupos foram formados: cluster 1 (azul escuro), composto por UFF, UFPE, UFSCar e Unicamp; cluster 2 (vermelho), formado pela PUC, UEM, UFPR, UFSC, UNIFESP e USP; cluster 3 (amarelo), composto por UERJ, UFBA e UFRJ; cluster 4 (verde), constituído por FIOCRUZ, UFJF, UFMG, UFPel e UFV; cluster 5 (lilás) estabelecido pela UFLA, UFRGS, UFRN; cluster 6 (azul claro) formado pela UFG e UNB. Nota-se uma predominância de instituições das regiões Sul e Sudeste, o que, possivelmente, indica uma articulação maior das organizações dessas regiões quando comparada com as demais. Também se observa uma participação de organizações das regiões Nordeste e Centro-Oeste e nenhuma participação do Norte. Sobre outra perspectiva, a parte inferior da Figura, mapa de densidade, mostra onde estão concentradas as colaborações. Percebem-se alguns pontos com maior adensamento; esses estão diretamente correlacionados à coautoria entre as instituições e o número de itens no entorno. Nesse mapa de calor se destacam a USP, UFRJ e UFMG, UFV e UFRGS. De forma geral, na Figura 6, é importante ressaltar que essa rede é construída com base nas coautorias, e não na quantidade absoluta de artigos. 


\section{ANÁLISE BIBLIOMÉTRICA SOBRE POLÍTICAS PÚBLICAS}

Figura 6 - Estrutura colaborativa do Brasil com base nas organizações

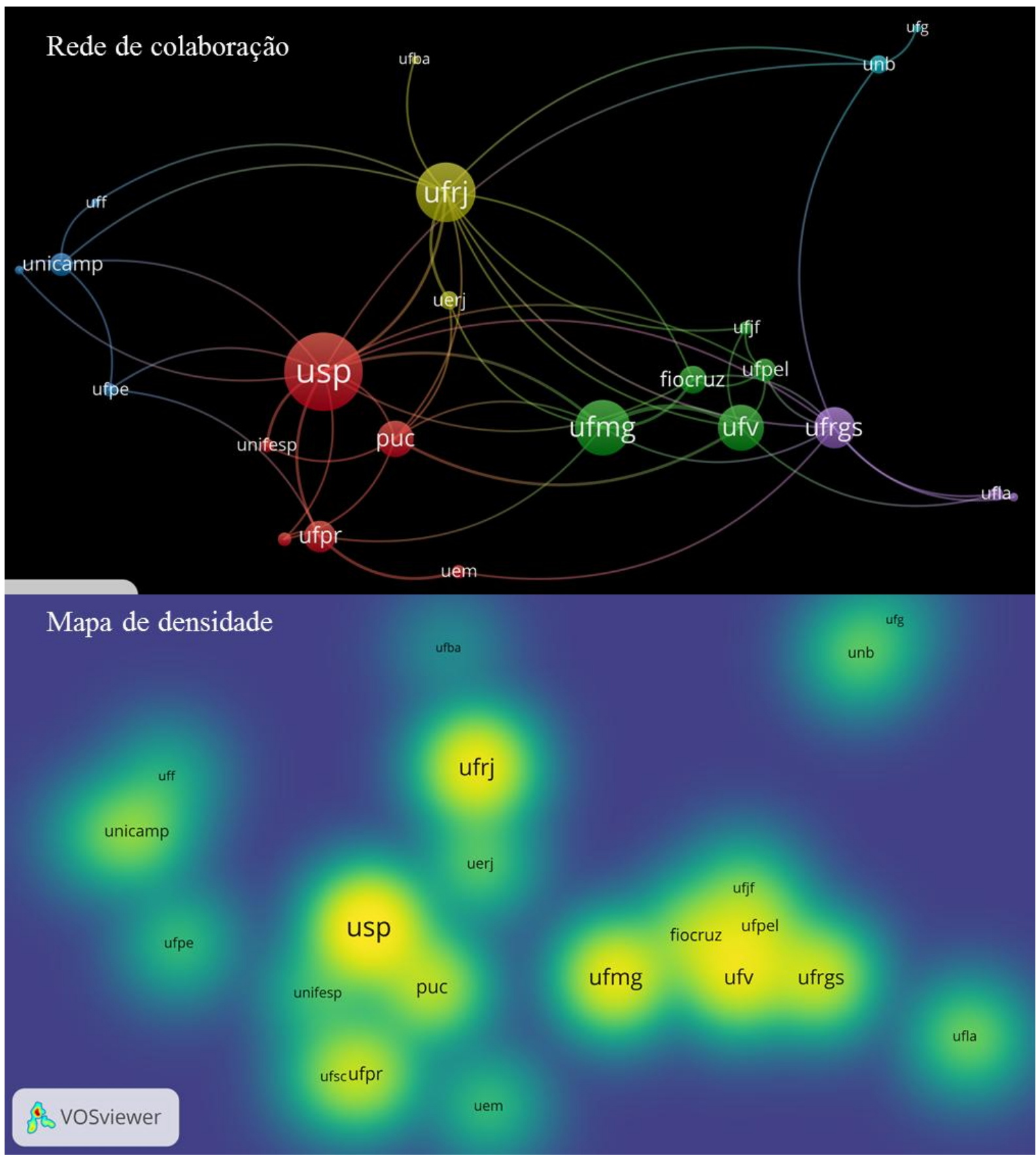

Fonte: Adaptado da base Scopus.

A Figura 7 mostra a rede bibliométrica formada pela análise de cocitação; nessa parte avaliou-se qual o grupo de autores são citados juntos. Os itens (769 autores) que compõem a rede foram selecionados com base: no número de citação, mínimo 30; e associação com outros autores, pelo menos uma conexão. A parte superior da Figura mostra a rede geral formada, e a inferior uma visualização aproximada do grupo vermelho. De forma geral, quanto mais próximo um autor estiver do outro, maior a chance de serem citados juntos independentemente do grupo no qual se encontram. Observa-se um total de oito grupos, cada um identificado por uma cor; autores de um mesmo grupo 
Fabio Freitas da Silva, Geísa Pereira Marcilio Nogueira, Ítalo de Oliveira Matias, Ludmila Gonçalves da Matta e Aldo Shimoya

têm uma probabilidade maior de serem citados em conjunto. Por exemplo, no grupo vermelho, quem citou Sousa também citou Avritzer, Arretche e Frey. Os autores desses clusters discutem, possivelmente, temas de pesquisa específicos dentro do contexto das políticas públicas; isso fica nítido nos grupos mais isolados com poucas conexões com outros grupos.

A Figura 8 também mostra a análise de cocitação, porém foi construído com base na densidade dos itens, áreas mais densas indicam a concentração das citações. Os autores que se destacaram por serem citados com outros pesquisadores foram: Laranjeira; Malta; Monteiro; Focault; e Souza. Outro ponto foram os grupos isolados que corroboram com a ideia de temas de pesquisa específicos.

Figura 7 - Análise de cocitação, autores que são citados juntos

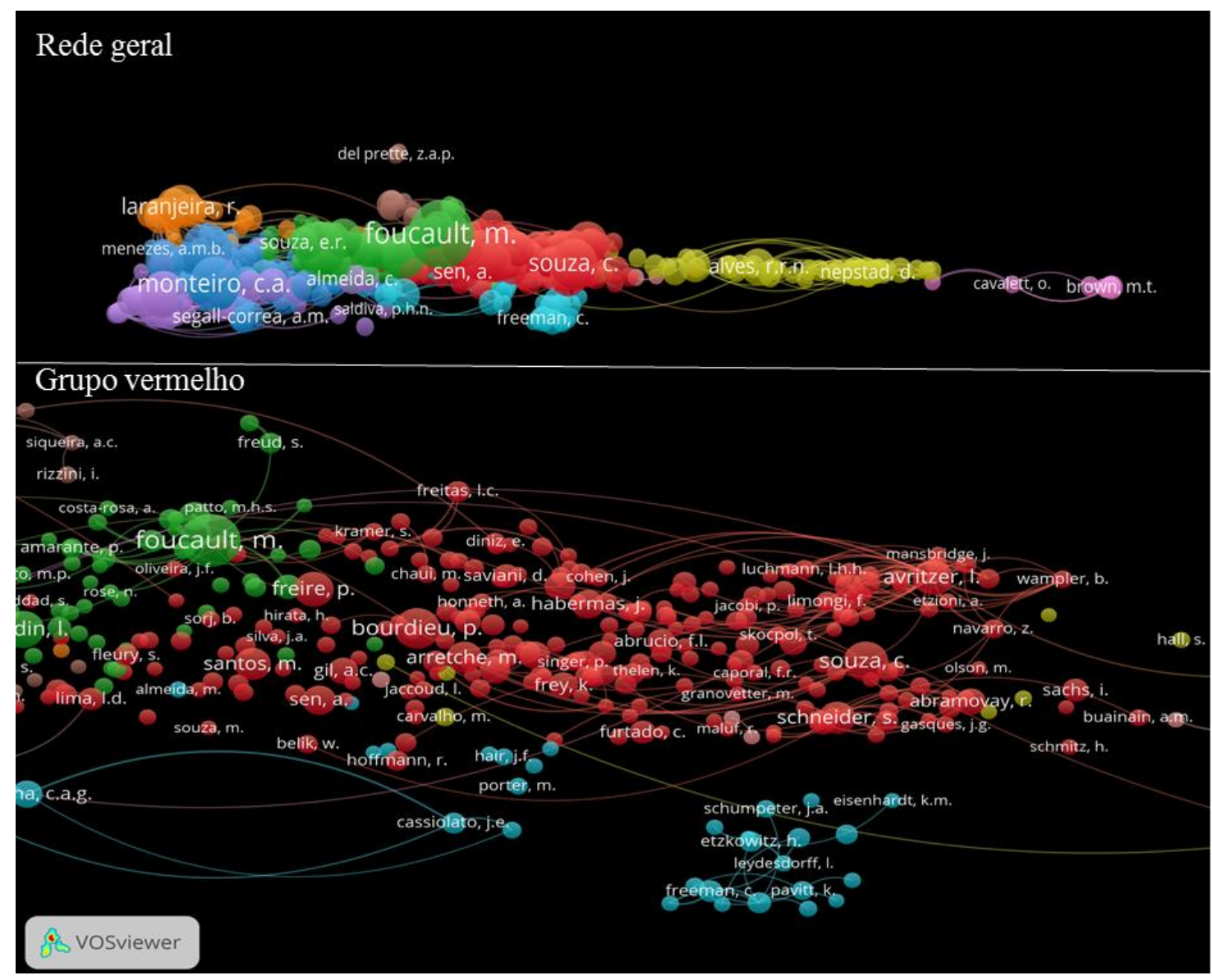

Fonte: Adaptado da base Scopus 


\section{ANÁLISE BIBLIOMÉTRICA SOBRE POLÍTICAS PÚBLICAS}

Figura 8 - Análise de cocitação por densidade, autores que são citados juntos

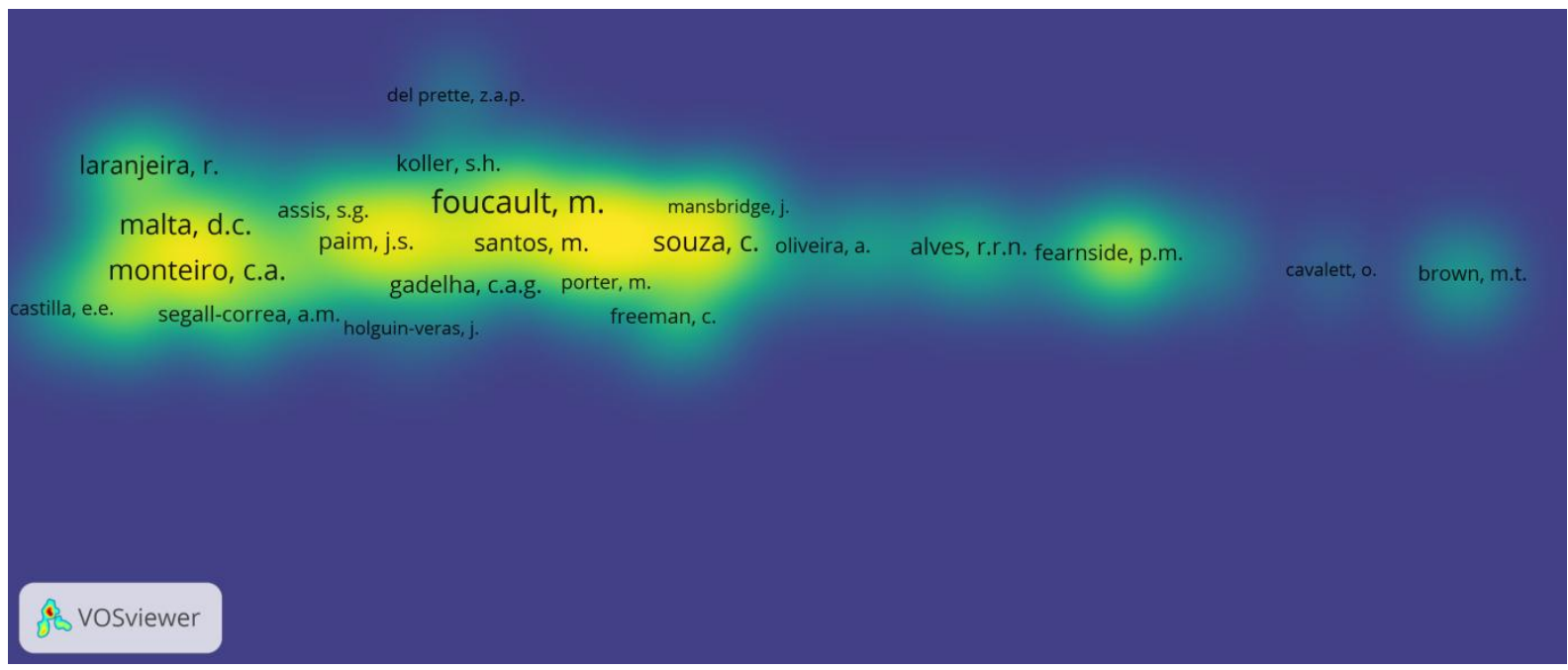

A Figura 9 mostra a análise de copalavra; esta avalia não só a frequência como também a co-ocorrência de um termo. Foram selecionadas as palavras-chave que apresentaram mais de 10 ocorrências, o que totalizou 193 palavras cada uma com no mínimo 1 ligação. Observaram-se cinco agrupamentos principais: vermelho, verde, amarelo, azul e lilás. Foi interessante notar que cada um desses clusters teve uma temática diferente; o vermelho foi caracterizado por palavras que remetem ao desenvolvimento sustentável, mudanças climáticas inovação, biocombustível e desenvolvimento rural. Por outro lado, o grupo verde aborda assuntos relacionados a avaliação, educação, desigualdade, desenvolvimento. Esses dois primeiros agrupamentos têm uma articulação com a palavra-chave política pública, porém em contextos diferentes, o primeiro conjunto está associado a "public policy" e o segundo a "public policies"; essas duas palavras têm conotações diferentes na língua inglesa apesar de em português diferirem somente no plural. O cluster lilás aborda questões como gênero, sexualidade, família, mulheres, violência, drogas, adolescência e juventude. 0 grupo amarelo pode ser caracterizado pelo debate de políticas públicas voltadas à saúde. $\mathrm{O}$ grupo azul demonstra duas temáticas diferentes: a primeira direcionada a cuidados na criança e adolescência com ênfase em assuntos sobre obesidade, nutrição e alimentação; a segunda tem um perfil voltado à qualidade de vida, fatores de risco, epidemiologia, fatores socioeconômicos, desigualdade na saúde, criança e mortalidade. 
Figura 9 - Análise de copalavra com base nas palavras-chave

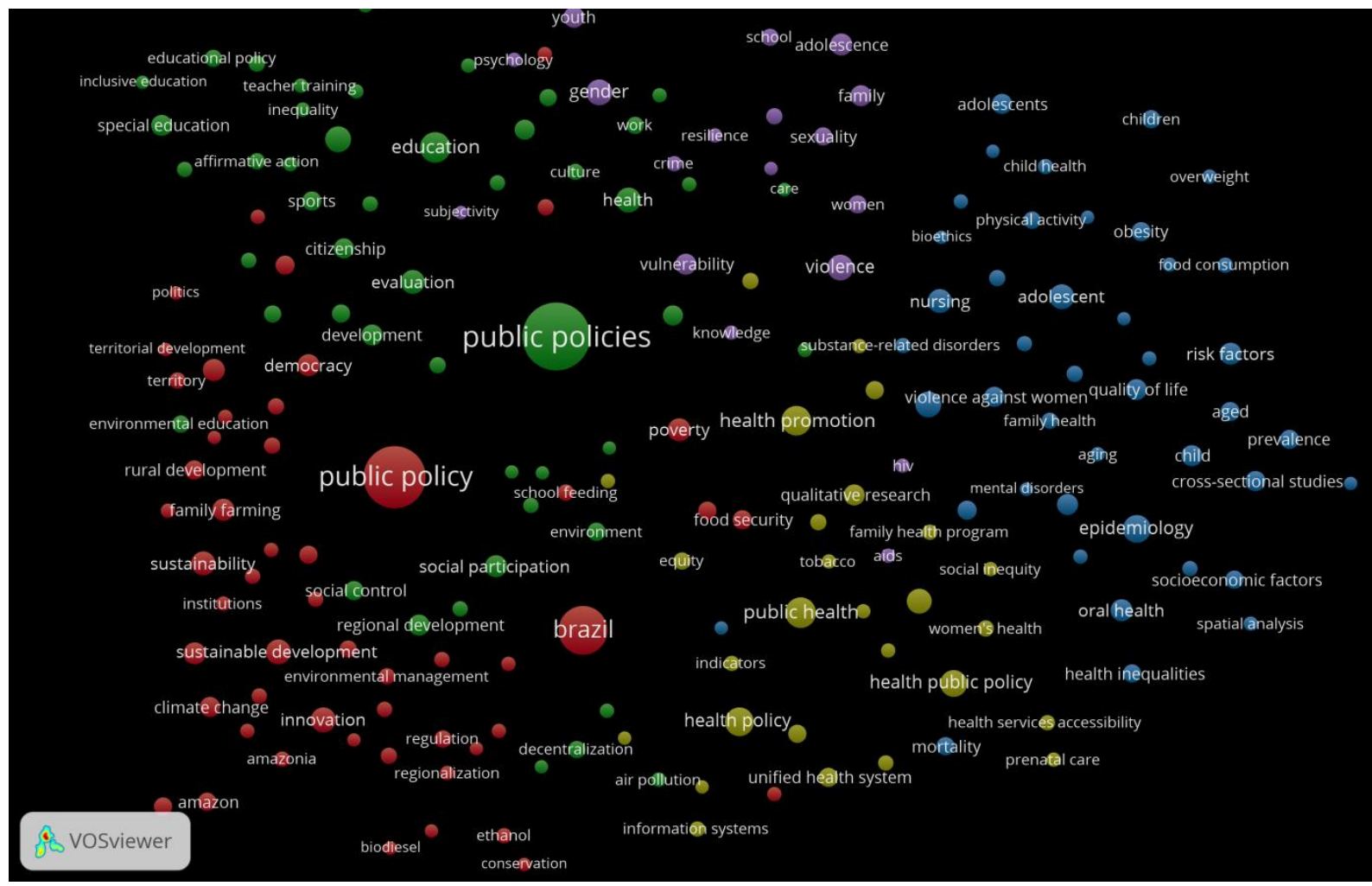

Fonte: Dados da base Scopus.

Na Figura 9 ainda podem ser exploradas as lacunas do conhecimento. Foi possível perceber pouca interação entre alguns termos, por exemplo, desenvolvimento regional ou desenvolvimento sustentável com qualidade de vida ou desigualdade na saúde. Assuntos localizados em lados opostos geralmente indicam pouco envolvimento.

\section{CONCLUSÃO}

O objetivo do presente trabalho foi realizar uma bibliometria sobre o tema políticas públicas. Como área de pesquisa e disciplina acadêmica, a política pública nasceu nos Estados Unidos; consequentemente, esse país liderou o número de publicações dessa disciplina com 23.214 trabalhos; já o Brasil apareceu em quarto lugar, com 4.005 publicações. A primeira discussão, no meio acadêmico, surgiu em 1909, nos Estados Unidos, enquanto, no Brasil, em 1987. Apesar da liderança dos EUA no volume de artigos, a organização com maior quantidade de estudos publicados foi uma instituição brasileira, USP. Globalmente, o tema política pública mostrou-se uma área multidisciplinar, com destaque para dois setores: Ciências Sociais e Medicina, que, juntos, somam $43 \%$ do total de produções cientificas. 


\section{ANÁLISE BIBLIOMÉTRICA SOBRE POLÍTICAS PÚBLICAS}

No que diz respeito ao Brasil, foi observado que a sua rede colaborativa com outros países mostrou um grande intercâmbio com várias nações do mundo, contudo, apresentou maior combinação de coautoria com Portugal, Espanha e França. Em relação às colaborações entre as instituições destacam-se a USP, UFRJ e UFMG, UFV e UFRGS. Na análise de cocitação, autores que são citados juntos, foi possível identificar 769 pesquisadores que tiveram no mínimo 30 citações e, pelo menos, uma conexão com outros autores. Observou-se um total de oito grupos, autores de um mesmo grupamento têm uma probabilidade maior de serem citados em conjunto. Por fim, na avaliação de copalavra foram analisadas a frequência e as conexões de palavras-chave dos artigos; notou-se que cada grupo de palavras abordava temáticas diferentes, além disso, podem-se perceber as lacunas de pesquisa do tema políticas públicas. Por exemplo, houve pouca interação entre as palavras desenvolvimento regional e desigualdade na saúde.

Vale lembrar que a bibliometria é uma técnica com viés quantitativo em uma análise subjetiva. Mostrou-se muito útil para fazer um mapeamento geral da ciência, bem como para definir um núcleo de leitura inicial. Contudo, apesar de a base de dados escolhida ser muito abrangente, isso não significa dizer que ela represente toda produção cientifica na área. Outro ponto importante colocado por alguns autores foi frisar que nada substitui a leitura extensiva dos trabalhos.

Como trabalho futuro, sugere-se uma revisão sistematiza da literatura, isto é, a leitura dos artigos para uma compreensão aprofundada das questões debatidas sobre o tema políticas públicas.

\section{REFERÊNCIAS}

BRONFENBRENNER, Urie. Ecology of the family as a context for human development: Research perspectives. Developmental psychology, v. 22, n. 6, p. 723, 1986.

CASTRO, Maria Helena Guimarães. Políticas Públicas: conceitos e conexões com a realidade brasileira. In: CANELA, Guilherme (org.). Políticas Públicas Sociais e os Desafios para o Jornalismo. São Paulo, SP: Cortez, 2008.

COBO, Manolo J. et al. An approach for detecting, quantifying, and visualizing the evolution of a research field: A practical application to the fuzzy sets theory field. Journal of Informetrics, v. 5, n. 1, p. 146-166, 2011.

EASTON, D. A Framework for Political Analysis. Englewood Cliffs: Prentice Hall. 1965.

FREY, Klaus. Políticas Públicas: um debate conceitual e reflexões referentes à prática da análise de políticas públicas no Brasil. Planejamento e Políticas Públicas. 2000.

EASTERLY, William; LEVINE, Ross. Africa's growth tragedy: policies and ethnic divisions. The quarterly journal of economics, v. 112, n. 4, p. 1203-1250, 1997. 
Fabio Freitas da Silva, Geísa Pereira Marcilio Nogueira, Ítalo de Oliveira Matias, Ludmila Gonçalves da Matta e Aldo Shimoya

GUEDES, Vânia L.S; BORSCHIVER, Suzana. Bibliometria: uma ferramenta estatística para a gestão da informação e do conhecimento em sistemas de informação, de comunicação e de avaliação científica e tecnológica. Encontro Nacional de Ciência da Informação, v. 6, p. 1-18, 2005.

KRAUT, Robert et al. Internet paradox: A social technology that reduces social involvement and psychological well-being?. American psychologist, v. 53, n. 9, p. 1017, 1998.

LASWELL, H.D. Politics: Who Gets What, When, How. Cleveland, Meridian Books.1936/1958.

LINDBLOM, Charles E. Still Muddling, Not Yet Through. Public Administation Review, p. 517-526. 1979.

LINDBLOM, Charles E. The Science of Muddling Through. Public Administration Review, p. 78-88. 1959.

LINK, Bruce G.; PHELAN, Jo. Social conditions as fundamental causes of disease. Journal of health and social behavior, p. 80-94, 1995.

MCLEROY, Kenneth R. et al. An ecological perspective on health promotion programs. Health education quarterly, v. 15, n. 4, p. 351-377, 1988.

MEYER, llan H. Prejudice, social stress, and mental health in lesbian, gay, and bisexual populations: conceptual issues and research evidence. Psychological bulletin, v. 129, n. 5, p. 674, 2003.

MOZAFFARIAN, Dariush et al. Executive summary: heart disease and stroke statistics-2015 update. Circulation, v. 131, n. 4, p. 434-441, 2015.

PERSSON, Olle; DANELL, Rickard; SCHNEIDER, J. Wiborg. How to use Bibexcel for various types of bibliometric analysis. Celebrating scholarly communication studies: A Festschrift for Olle Persson at his 60th Birthday, v. 5, p. 9-24, 2009.

PRITCHARD, A. Statistical bibliography or bibliometrics? Journal of Documentation: a Bibliometric Study, v. 25, n.4, p. 348-349, dec, 1969.

RAGAUSKAS, Arthur J. et al. The path forward for biofuels and biomaterials. Science, v. 311, n. 5760, p. 484-489, 2006.

RIMM, Eric B. et al. Vitamin E consumption and the risk of coronary heart disease in men. New England Journal of Medicine, v. 328, n. 20, p. 1450-1456, 1993.

SIMON, Herbert. Comportamento Administrativo. Rio de Janeiro: USAID. 1957.

SOUZA, Celina. Políticas públicas: uma revisão de literatura. Sociologias. Porto Alegre, v. 8, n. 16, p. 20-45. 2008.

ELSEVIER. Scopus. 2018. Disponível em:

https://www.elsevier.com/_data/assets/pdf_file/0008/208772/ACAD_R_SC_FS.pdf. Acesso em: 6 fev. 2019. 


\section{ANÁLISE BIBLIOMÉTRICA SOBRE POLÍTICAS PÚBLICAS}

TEECE, David J. Profiting from technological innovation: Implications for integration, collaboration, licensing and public policy. Research policy, v. 15, n. 6, p. 285-305, 1986.

TILMAN, David et al. Forecasting agriculturally driven global environmental change. science, v. 292, n. 5515, p. 281-284, 2001.

VAN ECK, Nees Jan; WALTMAN, Ludo. Software survey: VOSviewer, a computer program for bibliometric mapping. Scientometrics, v. 84, n. 2, p. 523-538, 2010.

VAN ECK, Nees Jan; WALTMAN, Ludo. Visualizing bibliometric networks. In: Measuring scholarly impact. Springer International Publishing, 2014. p. 285-320.

ZHAO, Qing; SADLER, B.m.. A Survey of Dynamic Spectrum Access. leee Signal Processing Magazine, v. 24, n. 3, p.79-89, 2007.

ZUPIC, Ivan; ČATER, Tomaž. Bibliometric methods in management and organization. Organizational Research Methods, v. 18, n. 3, p. 429-472, 2015. 